\title{
A NOVEL EMBEDDED SYSTEM DESIGN FOR AUTOMATIC TRAFFIC VIOLATION MONITORING AND CONTROL
}

\author{
Karthik R.P ${ }^{1}$, Swathi $K^{2}$, Ganavi $\mathbf{C ~ N}^{3}$, Prajwal $\mathbf{M}^{4}$ \\ ${ }^{1}$ Assistant Professor, Department of Electrical and Electronics Engineering, VVIET, Mysore, India-570028 \\ ${ }^{2}$ Assistant Professor, Department of Electrical and Electronics Engineering, VVIET, Mysore, India-570028 \\ ${ }^{3}$ Assistant Professor, Department of Electrical and Electronics Engineering, VVIET, Mysore, India-570028 \\ ${ }^{4}$ Application Development Analyst, Accenture, Bangalore, India - 560066
}

\begin{abstract}
This paper is mainly concerned about a system that has been premeditated to detect all traffic violations like over speeding, stop line violation, not stopping the vehicle during the red light phase of the traffic signal and then trace the individuals who have violated the traffic rules. To achieve the same two rows of Infrared transmitters are used. First one is to warn the driver during the first time of his violation, that is when is tries he move even though there is a red sign on the signal sensor displays the warning message to the driver asking him to stop for the red signal and move only when the signal has changed to green. Even the display of warning message if the driver continues to move without stopping their vehicle then the Second transmitter send a notification to the Road Traffic Officer (RTO) regarding the violation details and the car details that has violated the traffic rules and next time the cars stops and the engine is switched off, the engine gets auto locked and only Road traffic officer can open the lock and penalizing the violator. The designed system basically has 2 sections, first the transmitter that is mounted in the car and the second is the receiver that is at the Road traffic office. So the vehicle details are monitored and transmitted to the RTO section whenever the vehicle violates the traffic rule.
\end{abstract}

Keywords-GSM (Global System for Mobiles), RTO (Regional Transport Office), IR LED (Infrared Light Emitting Diode)

\section{INTRODUCTION}

Now a day's embedded system could be a part of our life and will be even a lot of current within the future. Most of them perform straight forward management and support tasks on command in a very pre-programmed manner; however the same has not been implemented when it comes to the area of road section. Increasingly they're designed to hold out autonomous tasks including "intelligence" (Smart Systems) they're conjointly designed to be able to decide, supported advanced inputs and situation awareness that is, what to try to do in circumstances that are defined by associate unreliable setting. Several security systems that begin from security lock codes to finger print scanner, iris scanner for security locks are a results of development within the field of embedded systems. Several safety systems like speed management in class vans, fireplace detection system and smoke detection systems in residences, laboratory, factories and entrant detection in protected areas are the most important embedded systems that are used wide around the world. Hence, these sorts of embedded systems play a significant role in safety and security systems. Designing autonomous vehicles are ensuing step during this direction of development within the space of embedded technology.

As we all know in the recent times, traffic accidents \& congestions have significantly increased. The vehicle volume has increased exponentially; the road infrastructure has not been improved proportionately. This successively results in enhanced traffic congestion and road accidents. Quite different technologies are there to find traffic jam and to reduce congestion. Management, addition economical requirements marks several drawbacks of this technologies which also include issues like installation issues, complexity, cost, etc. In a trial to cut back the issues related to traffic \& improve the traffic discipline, advanced technological solutions are projected through this paper. If a driver violates any of the traffic rules, the motive force is going to be charged in line with the RTO rules.

The drivers need to follow the Rules of the road and end up. These rules sometimes apply to any or all road users, though they are of special importance to cycle riders and motor bike riders. Interactions between vehicles and with pedestrians are checked by these rules. The essential traffic rules area unit outlined by Associate in Nursing international accord underneath the authority of the global organization, the 1968 Vienna Convention on Road Traffic. Not all countries area unit soul to the convention and, even among signatories, native variations in follow are also found. There are also unwritten native rules of the road, that area unit typically understood by native drivers. As a general rule, a driver is expected to avoid a collision with another vehicle and pedestrians, in spite of whether or not the applicable rules of the road enable them to be wherever they happen to be. 
Although there are additional principals that are applicable for various traffic moments, they are not being followed. So we need to create mistake proofing system since the rule and principals aren't being followed. And in recent they are many that have led to increase in the traffic and this increase also led to increase in the traffic issues like accidents and other loses because of traffic violation. Talking about the increase of traffic violations which is a threat to personal and public safety calls for a real time demand for building efficient systems that tracks the traffic violation. Large-scale machine-driven image social that are developing control technologies that have provided powerful tools that can curb traffic violation and also by switching unsafe driving behavior by educating communities that unsafe driving is going to be penalized.

In various developed countries like America, UK, Canada and Australia traffic cameras are installed which captures images of all the vehicles that are violating the traffic rules. To explain more about this traffic camera, it is a red light weight camera that is short for red light. The triggering process of the camera happens when a vehicle enters the intersection when the traffic signal has turned red. It automatically captures those motors that run over the red lights instead of stopping and the law enforcement officer can view the photo and consider this as a proof, penalize the violator by mailing the violation details to him and asking them to pay the penalized amount, warn them not to commit the same mistake again.

For a fast, well planned and cost effective management of traffic rules and violations, traffic violation management system is in place. The main basic plan for this system is the software which allows the user to track various administrative procedures of the Department of Transportation like the emission of notifications and fines. Security and amenities of road users are changing into serious worry. Due to the increase in the road accidents with increase in vehicle traffic, it is crucial to craft a safer and dependable arrangement for traffic control and management. The bull's eye for this journal is to present a system that can spot traffic violations like speed violation, lane violation, skipping the traffic signal and hence by tracing all the vehicles which have indulged in traffic violation.

\section{PROPOSED SYSTEM}

Fig 1 shows the overall scenario of proposed system which mainly includes Traffic signal, sensors used in vehicles and RTO. The proposed scheme in this project doesn't speak about the type of automobile used. Nevertheless, the implementation of the system to the automobiles is not restricted to four wheelers alone and is used globally. However, in this paper the installation of sensors in the vehicles is not discussed.

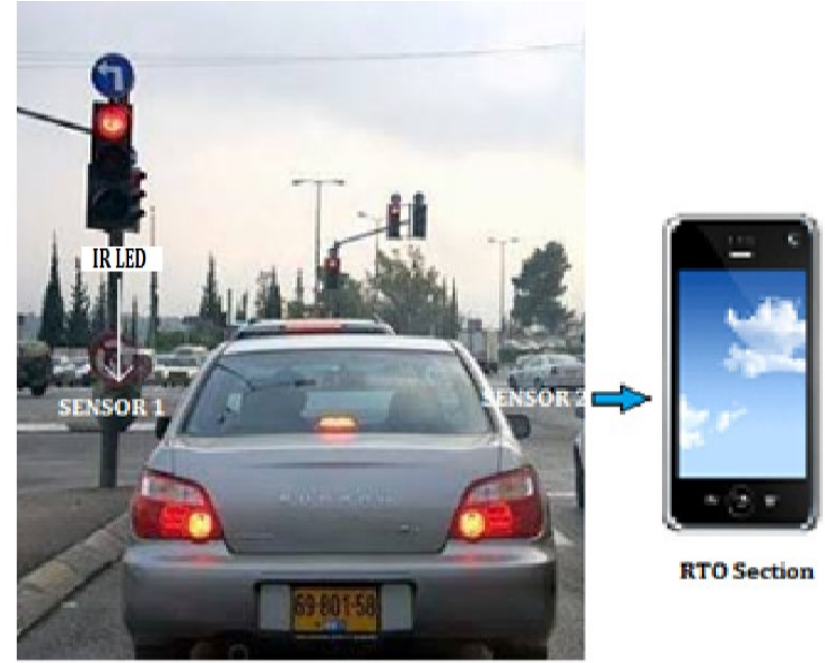

Fig-1 Proposed System

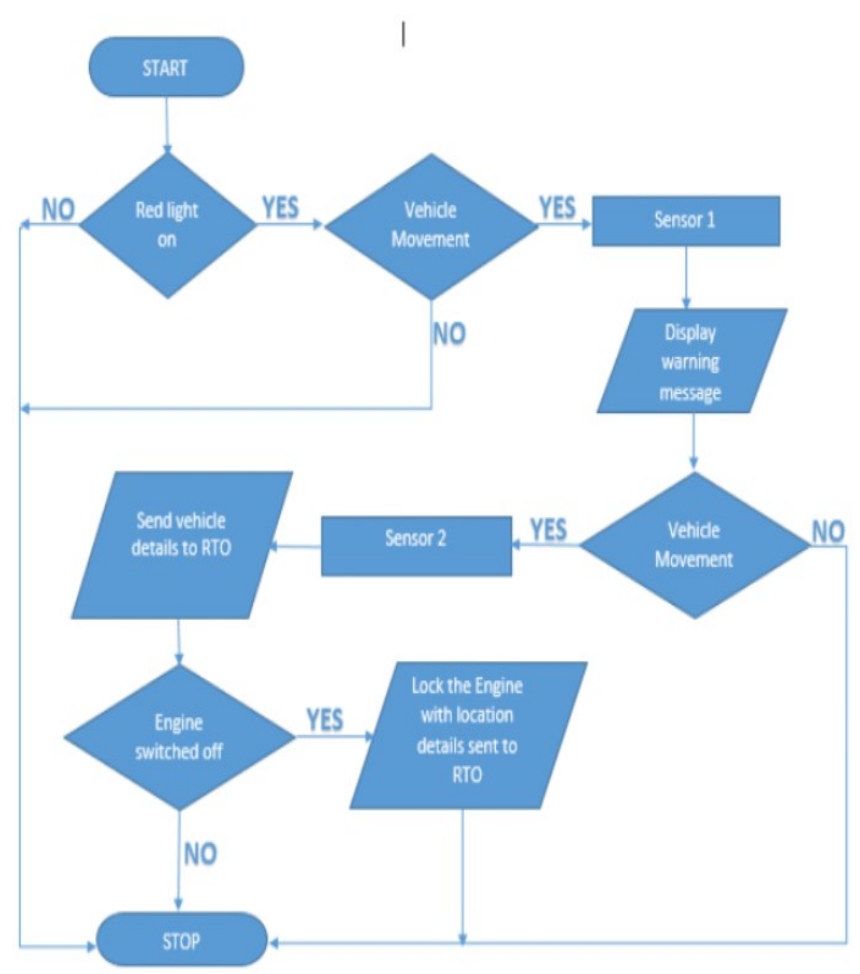

Fig-2: Flow chart

The flow chart of this process is as explained. When there is a red signal in the traffic, the first sensor in the car checks if the car is still moving even though there is a red signal on the traffic light .If yes then a warning message is displayed to the drive to stop the car and wait for the green signal.After the warning message is displayed the sensor checks if the car is moving and if the car is still in motion then the second sensor sends the vehicle and the owner details to the RTO office through GSM and engine is monitored in such a way that the next time it has been switched off ,the engine gets locked and the location and vehicle details is sent to RTO. The RTO themselves have to come to the vehicle location and unlock the engine and penalize the drivers for violating the traffic rules. 


\section{MODELING OF TRAFFIC SECTION}

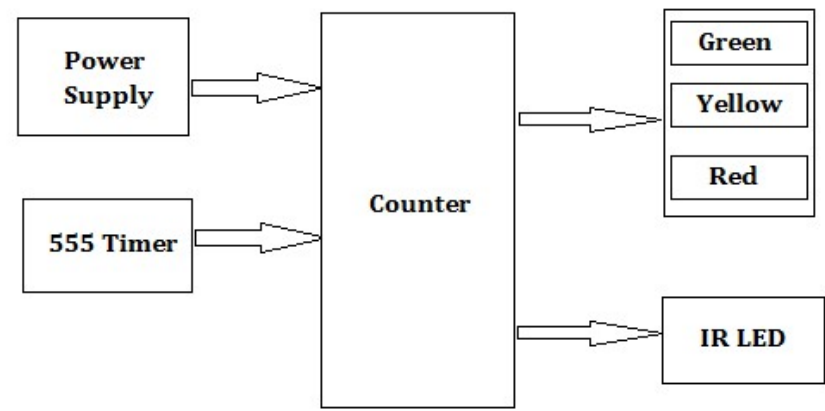

Fig-3: Block diagram of Traffic section

Traffic section consists of signals, timer, power supply, IR transmitter, and counter. Initially we give $5 \mathrm{v}$ power supply and set a delay between LED's using a 555 timer. Counter counts the delay between each LED's and IR radiations emitted by IR LED only when there is a red signal.

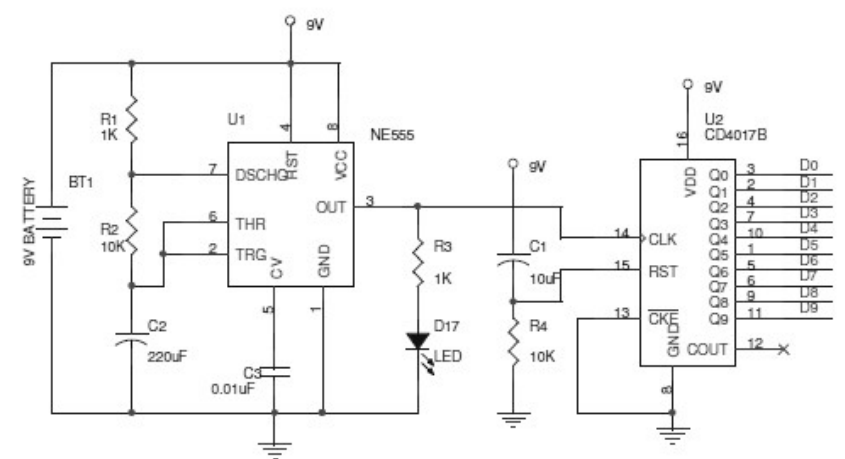

Fig-4: Circuit diagram of Traffic section

\section{MODELING OF VEHICLE SECTION}

Vehicle section consist of IR sensors, comparator, relay, buzzer, GSM module, power supply, Microcontroller, LCD, transistors. Vehicle section circuit have installed along with two sensors in a vehicle. When a first sensor cross the IR radiation emitted by LED during red signal comparator compares the input and gives the high output. it will warn a rider by displaying warning message.

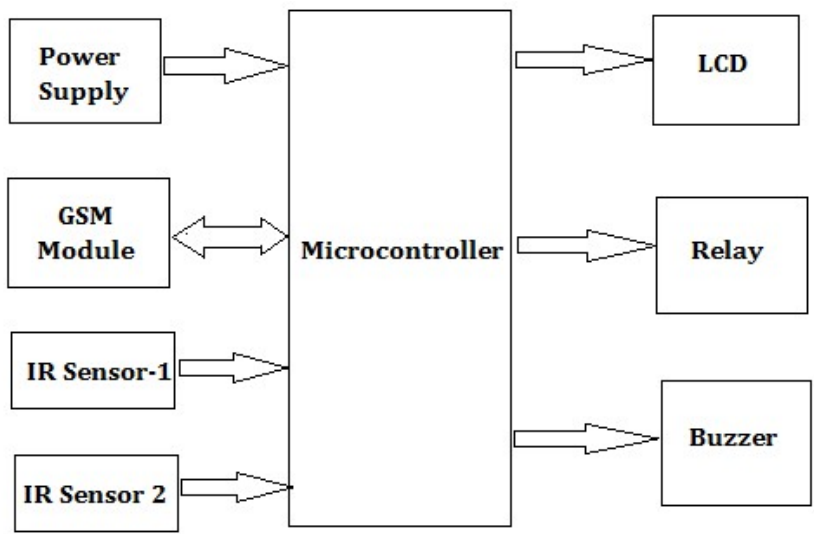

Fig-5 Block diagram of Vehicle section
If he further crosses the red signal microcontroller send a signal to relay and give a warning message to the rider. At the same time it transfer message to RTO section using GSM. After some delay it will turn off the ignition system.

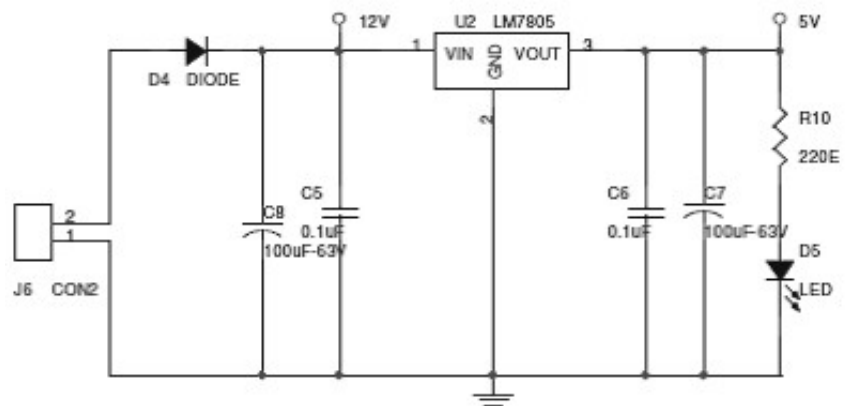

Fig-6: Circuit diagram of Vehicle section

\subsection{Modeling of RTO Section}

RTO section receives the message from vehicle section. It act's as server and it has the details of the driver. It has the power to turn on the ignition system after rider paid a penalty. Ignition system won't turn on until RTO officer turns it on.

\section{EXPERIMENTAL RESULTS}

Traffic section consists of signals, timer, power supply, IR transmitter, and counter. Initially we give $5 \mathrm{v}$ power supply and set a delay between LED's using a 555 timer. Counter counts the delay between each LED's and IR radiations emitted by IR LED only when there is a red signal.

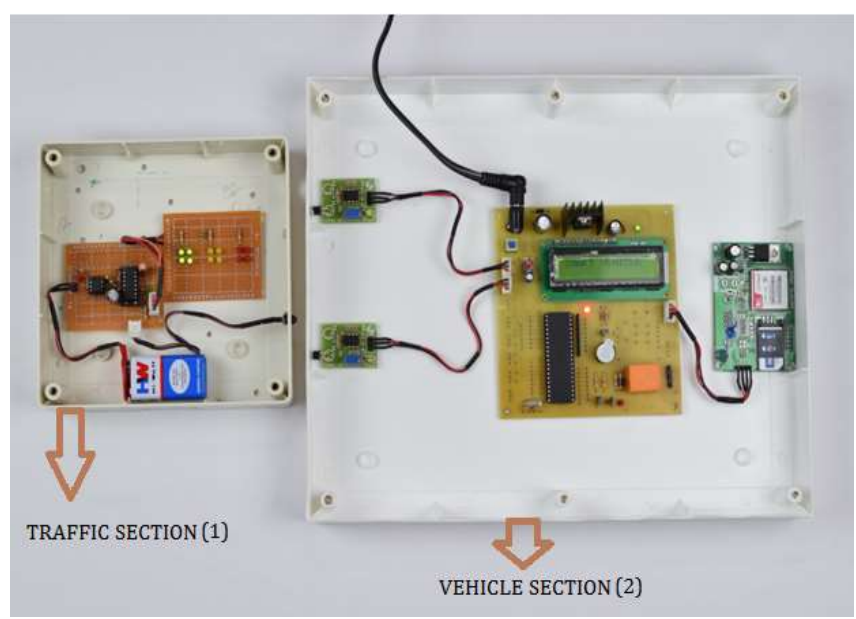

Fig-7: Hardware Implementation

Vehicle section consist of IR sensors, comparator, relay, buzzer, GSM module, power supply, Microcontroller, LCD, transistors. Vehicle section circuit have installed along with two sensors in a vehicle. When a first sensor cross the IR radiation emitted by LED during red signal comparator compares the input and gives the high output. it will warn a rider by displaying warning message. If he further crosses the red signal microcontroller send a signal to relay and give a warning message to the rider. At the same time it transfer message to RTO section using GSM. After some delay it will turn off the ignition system. 
RTO section receives the message from vehicle section. It act's as server and it has the details of the driver. It has the power to turn on the ignition system after rider paid a penalty. Ignition system won't turn on until RTO officer turn it on.

\section{CONCLUSION}

In this work, embedded system for automatic traffic violation monitoring, the traffic frequency is measured as a function of number of vehicles which comes under the contact of sensing unit. The implementation has a microcontroller based system that automatically performs the function of monitoring traffic violation using microcontroller. The advantage of this system goes by easy installation, strong function head and easy promotion. Future scope of this project is to employ a sophisticated system which has an ability to monitor more number of vehicles and send the violation message to the respective nearby traffic police or station in order to increase the efficiency and action on unlocking the automobile engine through GSM.

\section{REFERENCES}

[1]. Dhaval Shah, Sheth, Shival Trivedi and Shivang bakliwal, "microcontroller controller based traffic violation control system using wireless communication", IJECIERD, 2012, vol 2. pp.63-70.

[2]. G.S. Prasanth Ganes, B.Balaji and T.A. Srinivasa Varadhan, "anti - theft tracking system for automobiles", IEEE, 2011, pp.17-19.

[3]. V Ramya, B Palaniappan and M. Arul Jothi, "Embedded system for automatic traffic violation monitoring and altering", IJAIS, vol. 4, pp.26-33.

[4]. howstuffworks.com 